\section{(6) OPEN ACCESS}

\title{
What explains anorectal chlamydia infection in women? Implications of a mathematical model for test and treatment strategies
}

\author{
Janneke C M Heijne, ${ }^{1}$ Geneviève A F S van Liere, ${ }^{2,3}$ Christian J P A Hoebe, $^{2,3}$ \\ Johannes A Bogaards, ${ }^{1}$ Birgit H B van Benthem, ${ }^{1}$ Nicole H T M Dukers-Muijrers ${ }^{2,3}$
}

- Additional material is published online only. To view please visit the journal online (http://dx.doi.org/10.1136/ sextrans-2016-052786)

${ }^{1}$ Centre for Infectious Diseases Control, National Institute for Public Health and the Environment (RIVM), Bilthoven, The Netherlands

${ }^{2}$ Department of Sexual Health, Infectious Diseases and Environmental Health, South Limburg Public Health Service, Geleen, Netherlands ${ }^{3}$ Department of Medical Microbiology, Care and Public Health Research Institute (CAPHRI), Maastricht University Medical Center (MUMC+), Maastricht, Netherlands

Correspondence to Dr Janneke Heijne, National Institute of Public Health and the Environment, P. O. Box 1, Bilthoven $3720 \mathrm{BA}$, The Netherlands;

janneke.heijne@rivm.nl

Received 1 July 2016

Revised 3 November 2016 Accepted 28 November 2016

Published Online First

16 December 2016

CrossMark

To cite: Heijne JCM, van Liere GAFS, Hoebe CJPA, et al. Sex Transm Infect 2017:93:270-275
ABSTRACT

Objectives Female anorectal Chlamydia trachomatis (chlamydia) infections are common irrespective of recent anal sex. We explored the role of anorectal infections in chlamydia transmission and estimated the impact of interventions aimed at improved detection and treatment of anorectal infections.

Methods We developed a pair compartmental model of heterosexuals aged 15-29 years attending STI clinics, in which women can be susceptible to or infected with chlamydia urogenitally and/or anorectally and men urogenitally. Transmission probabilities per vaginal and anal sex act, together with an autoinoculation probability, were estimated by fitting to anatomic site-specific prevalence data ( $14 \%$ urogenital; $11 \%$ anorectal prevalence). We investigated the 10-year reduction in female chlamydia prevalence of interventions (universal anorectal testing of female STI clinic attendees or doxycycline use for urogenital chlamydia) relative to continued current care (anorectal testing on indication and doxycycline for anorectal and azithromycin for urogenital chlamydia).

Results The transmission probability per anal sex act was $5.8 \%$ (IQR 3.0-8.3\%), per vaginal sex act 2.0\% (IQR $1.7-2.2 \%)$ and the daily autoinoculation probability was $0.7 \%$ (IQR $0.5-1.0 \%)$. More anorectal chlamydia infections were caused by autoinoculation than by recent anal sex. Universal anorectal testing reduced population prevalence modestly with $8.7 \%$ (IQR 7.6-9.7\%), yet the reduction was double that of doxycycline use for urogenital infections (4.3\% (IQR 3.5-5.3\%)) relative to continued current care.

Conclusions Autoinoculation between anatomic sites in women might play a role in sustaining high chlamydia prevalence. A shift to more anorectal testing of female STI clinic attendees may be considered for its (albeit modest) impact on reducing prevalence.

\section{INTRODUCTION}

Chlamydia trachomatis (chlamydia) is the most commonly reported bacterial STI worldwide and, ${ }^{1}$ when untreated, can lead to serious long-term complications including pelvic inflammatory disease and infertility. As well as urogenital infections, chlamydia can also cause anorectal infections, with anorectal prevalences found between $5.6 \%$ and $17.5 \% .^{2}$ Women with anorectal chlamydia infection often have a concurrent urogenital chlamydia infection (71-95\%) and vice versa $(33-83 \%$ with a urogenital infection have a concurrent anorectal infection). ${ }^{2}$
Anorectal testing for chlamydia is often advised on indication in women with anal symptoms or recent anal sex. ${ }^{3-5}$ Studies on routine universal anorectal testing consistently found similar anorectal chlamydia prevalence in women with or without anal symptoms or history of anal sex..$^{6-8}$ This suggests that by testing on indication only, many anorectal chlamydia infections are missed. Furthermore, when women are infected at both locations, but not tested for anorectal infection, they receive the standard treatment for a urogenital infection, which is often azithromycin. ${ }^{3} 59$ A recent randomised controlled trial (RCT) found high efficacies for azithromycin (97\%) and doxycycline $(100 \%)$ in treating urogenital infections. ${ }^{10}$ However, there are no RCTs on the efficacy of azithromycin and doxycycline in clearing anorectal infections and a recent systematic review, based on observational studies, showed reduced effectiveness for treating anorectal chlamydia with azithromycin compared with doxycycline $(83 \%$ vs $99.6 \%){ }^{11}$ These findings suggest that many anorectal infections are suboptimally treated with the azithromycin provided for urogenital chlamydia, which could be a potential obstacle for controlling chlamydia transmission and its complications.

The high co-occurrence of anorectal and urogenital infections in women might be indicative of an autoinoculation process in which one anatomic site is inoculated with infective bodies from the other site. ${ }^{12}$ Taking autoinoculation into account, it has been calculated that the chance of a woman remaining free of urogenital chlamydia infection after treatment could be almost six times higher for doxycycline compared with azithromycin. ${ }^{13}$ However, the role of autoinoculation in chlamydia transmission has not yet been quantified.

Insights into the role of anorectal infections in chlamydia transmission and possible autoinoculation routes are needed for optimising testing and treatment guidelines. Mathematical modelling is a tool for understanding transmission of infections in a population and for estimating the impact of interventions. Here, the first aim is to develop a mathematical model to quantify the contribution of anorectal infections and autoinoculation in chlamydia transmission in women. The second aim is to estimate the population-level impact of interventions aimed at reducing anorectal chlamydia infections. 


\section{METHODS}

\section{Dynamic transmission model}

We used a deterministic pair compartmental model describing the pair formation and separation of heterosexual men and women aged 15-29 years attending STI clinics. The model is based on existing general population pair models. ${ }^{14}$ Here, the pair model was extended in two ways. First, two anatomical infection locations were incorporated for women: anorectal and urogenital location. Since anorectal infections among heterosexual men are scarce, ${ }^{2}$ only the male urogenital location was incorporated. Second, the model was extended to include two types of partnerships: one that includes anal sex as well as vaginal sex and one with vaginal sex only. The partnerships can be formed and broken up at any time. A detailed description of the model is given in the online supplementary text $\mathrm{S} 1$, and the parameters and their values are shown in table 1 and online supplementary tables S1 and S2.

In brief, at both anatomic locations, individuals can either be susceptible or infected. We assumed natural clearance of infection to be site-specific, meaning that women can clear the infection at one anatomical location but stay infected at the other anatomical location. After treatment, or after site-specific natural clearance, individuals can become susceptible again. There are three ways of becoming infected. First, a transmission probability per vaginal sex act is included, leading to urogenital infections in both men and women. In order to confine the number of unknown parameters, we assumed the same probability for male-to-female as female-to-male transmission. Second, a transmission probability per anal sex act is included, leading to anorectal infections in women and urogenital infections in men, again with the same probability for transmission in either direction. Third, for women only, an autoinoculation process is included as a daily probability that an urogenital infection leads to an anorectal infection or that an anorectal infection leads to an urogenital infection, with the same probability in either direction.

\section{Empirical data}

We used data from female STI clinic visitors attending the South Limburg Public Health Service between May 2012 and July 2013, where patients aged 18 years and older were offered routine universal chlamydia testing on both anatomical locations. ${ }^{6}$ Samples were tested for chlamydia using nucleic acid amplification assays (NAAT). We used data from all heterosexual females aged $18-29$ years $(n=434)$ excluding sex workers or swingers to obtain point estimates of urogenital, anorectal and concurrent (urogenital and anorectal) infection prevalence and 95\% CIs and to obtain the percentage reporting anal sex. All other behavioural parameters are from all STI clinic attendees aged 15-29 years or other relevant sources.

\section{Calibration procedure}

The three transmission probabilities (per vaginal and anal sex act transmission probability and autoinoculation probability) were calibrated to the STI clinic data assuming a background female testing uptake of $14 \%$ per year, ${ }^{15}$ a partner notification probability of $0.5^{16}$ and the Dutch standard of care. ${ }^{17}$ In this standard of care, all STI clinic attendees receive urogenital chlamydia testing and only women who reported having had anal sex in the six months prior to their clinic visit are also tested

Table 1 Parameters of the model and corresponding values reflecting a heterosexual population of men and women aged 15-29 years visiting STI clinics (excluding swingers and prostitutes) receiving the standard of care

\begin{tabular}{|c|c|c|}
\hline Parameter & Baseline value & Source \\
\hline \multicolumn{3}{|l|}{ Infection } \\
\hline Duration of untreated urogenital infection (males and females), months & 12 & 27 \\
\hline Duration of untreated anorectal infection (females), months & 12 & 28 \\
\hline \multicolumn{3}{|l|}{ Transmission } \\
\hline Transmission probability per vaginal sex act & Calibrated & \\
\hline Transmission probability per anal sex act & Calibrated & \\
\hline Daily autoinoculation probability & Calibrated & \\
\hline \multicolumn{3}{|l|}{ Behaviour } \\
\hline Frequency of unprotected vaginal sex acts, per week & 2 & 29 \\
\hline Frequency of unprotected anal sex, per week & 0.5 & $29 *$ \\
\hline Number of partners, per yeart & 3 & STI clinic \\
\hline Fraction of people in a partnership at any time, $\% \dagger$ & 66 & STI clinic \\
\hline Anal sex past six months, \%§ & 18 & STI clinicq \\
\hline \multicolumn{3}{|l|}{ Intervention parameters } \\
\hline Azithromycin effectiveness ${ }^{* *}$ urogenital infection, $\%$ & 94.3 & 30 \\
\hline Doxycycline effectiveness ${ }^{* *}$ urogenital infection, \% & 97.1 & 30 \\
\hline Azithromycin effectiveness** anorectal infection, \% & 82.9 & 11 \\
\hline Doxycycline effectiveness ${ }^{* *}$ anorectal infection, \% & 99.6 & 11 \\
\hline Female testing uptake, \% per year & 14 & 15 \\
\hline Period of recent anal sex to determine which treatment to provide, months & 6 & 17 \\
\hline Partner notification probability & 0.5 & 16 \\
\hline
\end{tabular}


anorectally. For chlamydia treatment, a single dose of azithromycin $(1 \mathrm{~g})$ is prescribed for urogenital infections, and 7-day (100 mg twice daily) use of doxycycline for anorectal infections when detected by testing.

Details of the calibration procedure can be found in the online supplementary text S2. Only sets of transmission probabilities that resulted in model point prevalences that fell within the $95 \%$ CIs of the data are used throughout the paper. For each of these parameter sets, we counted the cumulative number of events that resulted in transmission between individuals and within one individual between anatomical sites. We then calculated the distribution of events per anatomical location by dividing the cumulative number of infections caused by vaginal intercourse or by autoinoculation from the anorectal to the urogenital site by the total cumulative number of urogenital infections. For the anorectal location, we divided the cumulative number of infections caused by anal intercourse or by autoinoculation from the urogenital to the anorectal site by the total cumulative number of anorectal infections (see online supplementary text $\mathrm{S} 1$ ). All results are provided as medians and IQRs.

\section{Intervention scenarios}

We tested the impact of three different intervention scenarios (table 2). The impact is defined as the relative difference in chlamydia prevalence of female STI clinic visitors 10 years after introducing the intervention compared with continuation of the standard of care. First, we estimated the impact of universal doxycycline use, meaning that all people testing positive for urogenital chlamydia receive doxycycline. Second, we estimated the impact of universal testing on both anatomic sites where single urogenital infections were treated with azithromycin and anorectal infections were treated with doxycycline. Last, we tested the impact of both interventions combined.

\section{Uncertainty analyses}

We performed uncertainty analyses on the impact of the intervention for various parameters including the anorectal infection duration, the anal sex frequency, the percentage of people with recent anal sex to account for possible under-reporting, and the azithromycin and doxycycline effectiveness in treating anorectal and urogenital infections. We also looked at a scenario in which the standard of care for treating anorectal infections was azithromycin (eg, in the USA ${ }^{4}$ ) and a scenario with higher testing uptake (increased from $14 \%$ to $40 \%$ in 10 years as obtained in England). Last, we fitted the model to two other data sources on universal testing (see online supplementary text S3 and figure S2). ${ }^{7} 8$ For every uncertainty analyses, we refitted the model to the data and analysed the impact of interventions using the transmission probabilities that agreed with the data.

\section{RESULTS}

The model was able to produce chlamydia prevalence in agreement with the Dutch STI clinic visitor data (figure 1A). The anal infection prevalence in women was slightly higher in the model compared with the data, but still well within the CIs of the data.

\section{Quantifying transmission}

We found a median transmission probability of $2.0 \%$ (IQR $1.7-$ $2.2 \%)$ per vaginal sex act from men to women and women to men (figure 1B). The anal sex probability was more than twice that of vaginal sex, that is, $5.8 \%$ (IQR $3.0-8.3 \%$ ) from men to women and women to men. The female median daily autoinoculation probability was lower $(0.7 \%$; IQR $0.5-1.0 \%)$ than the per sex act transmission probabilities.

Using the calibrated transmission probabilities, we calculated the proportion of infections in females that were caused by intercourse or autoinoculation per anatomical location (figure $1 \mathrm{C})$. In the model, more urogenital infections were caused by vaginal sex (57\%; IQR 46-64\%) than by autoinoculation (43\%; IQR 36-54\%). The contribution of autoinoculation in anorectal infections was much larger, that is, 87\% (IQR 81-93\%) compared with $13 \%$ (IQR 7-18\%) through anal sex.

\section{Impact intervention scenarios}

The impact of the interventions is calculated as the relative reduction in female STI clinic visitors prevalence compared with continuation of the standard of care (see online supplementary figure S1). Introducing doxycycline as the universal treatment for all detected chlamydia infections had only a modest relative impact on reducing the total (urogenital and/or anorectal) chlamydia prevalence in the female STI clinic visitors population: the prevalence was reduced by $4.3 \%$ (IQR $3.5-$ $5.3 \%$ ) after 10 years compared with continuation of the standard of care in these 10 years (table 3 ). In absolute terms, this means that the total chlamydia prevalence in this STI clinic population (15.5\%; IQR 16.7-18.2\%) was only slightly reduced by $0.7 \%$ (IQR $0.6-0.9 \%$ ). Introducing routine universal testing on both anatomic locations had almost double the impact on reducing chlamydia prevalence (a relative reduction of $8.7 \%$ (IQR 7.6-9.7\%) after 10 years). In absolute terms, this reduction was small, with an absolute reduction of the prevalence of $1.5 \%$ (IQR 1.3-1.6\%). Both interventions combined resulted in the largest relative impact $(9.3 \%$; IQR $8.2 \% 10.3 \%)$ after 10 years, but the additional impact compared with universal testing alone was negligible. For all interventions, the relative reduction was slightly larger in anorectal prevalence compared with urogenital prevalence since the intervention measures were aimed at enhancing anorectal detection and treatment (see online supplementary tables S3 and S4).

Table 2 The standard of care that is incorporated in the model and the three studied intervention scenarios

\begin{tabular}{lllll}
\hline & $\begin{array}{l}\text { Standard of care } \\
35917\end{array}$ & $\begin{array}{l}\text { Universal routine } \\
\text { doxycycline }\end{array}$ & $\begin{array}{l}\text { Universal routine } \\
\text { anorectal testing }\end{array}$ & $\begin{array}{c}\text { Universal routine doxycycline } \\
\text { and anorectal testing }\end{array}$ \\
\hline $\begin{array}{l}\text { Testing } \\
\text { Urogenital location }\end{array}$ & Yes & Yes & Yes & Yes \\
$\begin{array}{c}\text { Anorectal location } \\
\begin{array}{l}\text { Treatment } \\
\text { Urogenital infection }\end{array} \\
\text { Anorectal infection }\end{array}$ & On indication & On indication & Yes & Yes \\
\hline
\end{tabular}



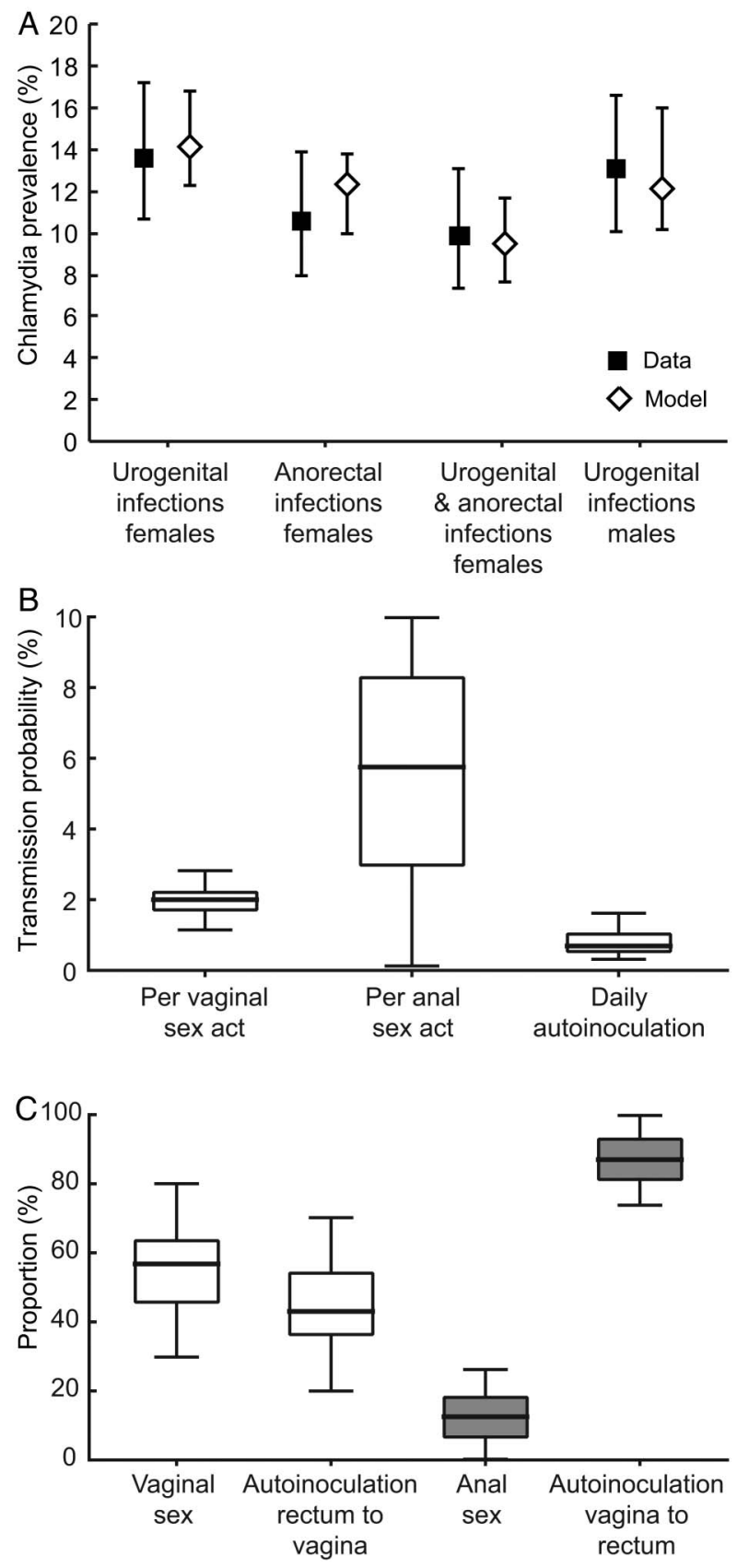

Figure 1 (A) Chlamydia urogenital, anorectal and concurrent infection prevalence in females and urogenital prevalence in males and $95 \% \mathrm{Cl}$ among heterosexuals aged $18-29$ years (excluding swingers and commercial sex workers) visiting the STI clinic in South Limburg, the Netherlands, between May 2012 and July 2013 (squares) and the calibrated median prevalence from the model (open diamonds) and the $95 \%$ percentiles of all runs. (B) The calibrated transmission probabilities for the three different transmission routes. (C) The distribution of female urogenital infections (white) and anorectal infections (grey) that occur through sexual intercourse or through autoinoculation. In (B) and (C), the results are presented as medians (black line), with the boxes representing the IQRs and the bars the minimum and maximum values.

\section{Uncertainty analyses}

The estimated impact of the intervention measures in reducing population prevalence was robust for the tested assumptions (table 3; see online supplementary tables S3 and S4). However, a larger reduction in population prevalence was estimated when assuming a lower azithromycin effectiveness in clearing anorectal infections and when azithromycin was the standard of care for treating anorectal infections. Additionally, for universal testing only, a larger reduction in prevalence was estimated when assuming a longer anorectal infection duration.

\section{DISCUSSION}

Our results suggest that autoinoculation between the female urogenital and anorectal location might play an important role in chlamydia transmission and in sustaining high chlamydia prevalence in women. While chlamydia is about equally prevalent at both the urogenital and the anorectal site, we showed that urogenital infections were much more due to vaginal sex than anorectal infections were to anal sex. This suggests that most infections in women start at the urogenital location through vaginal sex and are then transmitted to the anorectal site through autoinoculation. We also showed that, in a setting where doxycycline is provided for anorectal infections, adding routine universal anorectal testing of women was more effective in reducing STI clinic female prevalence compared with universal doxycycline use for urogenital and anorectal infections.

To our knowledge, we are the first to incorporate anatomic site-specific chlamydia infections in heterosexual population models. A strength of the model is that it incorporates partnership duration explicitly, meaning that reinfection from an infected partner within partnerships can occur. Another strength is that the model was validated using empirical data, enhancing the usefulness for policymaking. The results were robust across data sources from different settings and for most deviations in the assumptions. There are also limitations to the study. First, the model did not incorporate other possibilities than sexual exposure or autoinoculation on how people might become infected at the anorectal site such as via the gastrointestinal tract through oral sex (as suggested in animal models). ${ }^{12}$ Second, we assumed that all anorectal infections were also infectious. Current diagnostics for chlamydia (NAAT) cannot distinguish between live and dead organisms to indicate possible infectiousness. Bacterial load may provide some clues on this, and a recent study found that anorectal chlamydia load was within a similar range in women and men who have sex with men (MSM). ${ }^{18}$ Last, since the model is fitted to STI clinic data, it contains one group that is characterised by relatively high-risk sexual behaviour and high chlamydia prevalence compared with the general population. We speculate that adding more heterogeneity in the model (ie, by including low-risk individuals or allowing for dispersion of high-risk behaviour among STI clinic visitors) would estimate a smaller impact of the interventions on reducing prevalence. ${ }^{19}$

We incorporated site-specific natural clearance of the infections rather than individual-level clearance of infection. This means that when a woman is infected at both anatomical locations, she might clear the infection at one location, but not necessarily at the other. She would then be able to reinfect herself, thereby prolonging her infectious period, ${ }^{20}$ which might have led to an overestimation of the autoinoculation probability. While the possibility of site-specific clearance for chlamydia is still unknown, our assumption is in line with a previous modelling study looking at the role of site-specific infections in gonorrhoea transmission. ${ }^{21}$ Furthermore, a study on human papillomavirus acquisition and clearance among MSM estimated different clearance rates between the anorectal and urogenital locations, suggesting that individual-level clearance is not necessary. $^{22}$

The transmission probability per vaginal sex act found here was lower than that found in previous chlamydia models. Most 
Table 3 Relative reduction of total (urogenital and/or anorectal) chlamydia prevalence of female STI clinic visitors 10 years after introducing universal routine doxycycline treatment or universal routine anorectal testing or both interventions combined compared with continuation of standard of care

\begin{tabular}{|c|c|c|c|}
\hline & $\begin{array}{l}\text { Universal routine } \\
\text { doxycycline, \% (IQR) }\end{array}$ & $\begin{array}{l}\text { Universal routine anorectal } \\
\text { testing, \% (IQR) }\end{array}$ & $\begin{array}{l}\text { Both interventions } \\
\text { combined, \% (IQR) }\end{array}$ \\
\hline Baseline & $4.3(3.5-5.3)$ & $8.7(7.6-9.7)$ & $9.3(8.2-10.3)$ \\
\hline \multicolumn{4}{|l|}{ Uncertainty analyses } \\
\hline \multicolumn{4}{|l|}{ Infection parameters } \\
\hline Anal infection duration doubled ( 2 years) & $4.3(3.4-4.8)$ & $12.5(11.9-13.3)$ & $13.0(11.9-13.8)$ \\
\hline Anal infection duration halved ( 0.5 year) & $4.7(3.8-5.3)$ & $6.7(5.6-7.4)$ & $7.3(6.3-8.0)$ \\
\hline \multicolumn{4}{|l|}{ Behavioural parameters } \\
\hline Higher frequency of anal sex acts (once per week) & $4.3(3.4-4.9)$ & $8.3(7.6-9.1)$ & $8.8(8.2-9.7)$ \\
\hline $\begin{array}{l}\text { Lower vaginal sex acts in pairs that also engage in anal sex (same amount } \\
\text { of sex acts in both partnerships) }\end{array}$ & $4.5(3.9-5.3)$ & $9.0(8.1-10.0)$ & $9.6(8.8-10.5)$ \\
\hline Higher fraction of people with recent anal sex $(30 \%)$ & $2.8(2.3-3.1)$ & $5.2(4.6-5.6)$ & $5.8(5.3-6.1)$ \\
\hline \multicolumn{4}{|l|}{ Treatment parameters } \\
\hline Lower azithromycin effectiveness for anorectal infection (56\%) & $9.0(7.2-11.6)$ & $13.4(11.2-16.0)$ & $14.0(11.8-16.5)$ \\
\hline Lower doxycycline effectiveness for anorectal infection $(90 \%)$ & $2.5(2.1-2.5)$ & $6.7(5.9-7.1)$ & $7.2(6.5-7.7)$ \\
\hline Higher azithromycin and doxycycline efficacy for urogenital infection $(96.8 \% \text { and } 100 \%)^{10}$ & $4.6(3.8-5.5)$ & $8.9(7.8-9.7)$ & $9.5(8.6-10.4)$ \\
\hline \multicolumn{4}{|l|}{ Other scenarios } \\
\hline Azithromycin as the standard of care for anorectal infections ${ }^{4}$ & $7.0(6.0-8.5)$ & $11.4(10.1-12.9)$ & $12.1(10.7-13.4)$ \\
\hline Higher female testing uptake (from $14 \%$ to $40 \%$ per year) & $4.8(4.1-5.7)$ & $9.4(8.6-10.0)$ & $10.0(9.3-10.5)$ \\
\hline \multicolumn{4}{|l|}{ Other data source } \\
\hline Peters et al (South-African primary care facility data) $)^{8}$ & $3.2(2.7-3.7)$ & $5.9(4.4-7.3)$ & $7.3(5.9-8.6)$ \\
\hline Østergaard et al (Danish STI clinic data) $)^{7}$ & $4.4(3.6-5.0)$ & $9.1(7.6-9.9)$ & $9.8(8.5-10.5)$ \\
\hline
\end{tabular}

pair models estimated transmission probabilities between $6 \%$ and $17 \%{ }^{14}{ }^{23}$ compared with $2 \%$ in our study. This can be explained by the extra force of infection from the autoinoculation process in the current model, where the probability of becoming vaginally infected can be seen as a combination of both probabilities. Furthermore, compared with the other models, we assumed more sex acts per week (since we model STI clinic visitors) and no duration of immunity, which results, with equal duration of infection, automatically in a lower probability of transmission per vaginal sex act. Our weekly probability of becoming infected either by vaginal sex or autoinoculation was $9 \%$.

Our results are suggestive of a chlamydia autoinoculation process between anatomical sites in women. Support for an autoinoculation theory mainly comes from observational studies since hard evidence for this theory is lacking. For example, several studies found high anorectal detection rates in women not reporting anal intercourse. ${ }^{2}$ It is unknown whether other routes of transmission than autoinoculation could in part explain anorectal chlamydia detection. A recent study found that the anal use of fingers or toys was not associated with anorectal chlamydia detection, making this route of transmission less likely. ${ }^{6}$ Further observational evidence for autoinoculation processes comes from urinary tract infections, where bacteria that have their origin in the intestines invaded the urinary system. ${ }^{24}$ Moreover, our results that a large proportion (43\%) of chlamydia infections at the urogenital site were caused by autoinoculation are also in line with studies showing high repeated urogenital chlamydia infection rates among women. ${ }^{14} 25$

We estimated the relative impact of routine universal testing on reducing chlamydia prevalence to be almost double that of universal doxycycline treatment. An important factor that influences the impact of universal anorectal testing is the proportion of single anal infections. There is uncertainty around the prevalence of single anal infections in women, resulting in published estimates ranging between $4 \%$ and $25 \% .^{2}$ In the model, the anorectal prevalence is slightly higher than the data (but still within the CIs), resulting in a slightly higher percentage of single anorectal infections (22\%). However, this is well within the ranges published in the literature. ${ }^{2}$ Moreover, it is questionable if universal anorectal testing is feasible in practice since it would be costly to test all female STI clinic visitors on both anatomic locations. Future studies should include economic evaluations of the clinical impact of the interventions aimed at anorectal infections, taking into account the possibility that long-term complications such as infertility and ectopic pregnancies are averted by interrupting autoinoculation and thereby preventing repeated urogenital infections.

There is ongoing debate about the type of treatment for urogenital chlamydia infections, but a recent RCT did not find evidence that azithromycin was non-inferior to doxycycline in treating urogenital chlamydia. ${ }^{10}$ We showed that introducing doxycycline as the standard treatment for urogenital chlamydia only slightly reduced chlamydia prevalence in STI clinic visitors compared with continuation of azithromycin. It should be noted that the limited reduction in chlamydia prevalence was found when anorectal infections were already treated with doxycycline in the standard of care. When azithromycin is the standard of care for treating anorectal infections (as in the USA), ${ }^{4}$ a larger impact on reducing prevalence by introducing doxycycline treatment is expected. In both cases, introducing doxycycline as the standard treatment for chlamydia infections might still be beneficial on the individual level by preventing complications or the development of antimicrobial resistance against Mycoplasma genitalium. ${ }^{26}$ The largest impact of universal doxycycline treatment on reducing chlamydia prevalence was found for low values of the azithromycin effectiveness in clearing anorectal infections, highlighting the need for RCTs to determine treatment efficacies for anorectal chlamydia. 
In conclusion, autoinoculation between anatomic sites in women seems likely and could be important for continuing chlamydia transmission. Continued doxycycline use for anorectal chlamydia, together with a shift to more anorectal testing of female STI clinic attendees, may be considered for its (albeit modest) impact on reducing the continuously high-population chlamydia prevalence.

\section{Key messages}

- Studies on routine universal testing show that anorectal infections in women are common, irrespective of reported anal intercourse.

- The mathematical model suggests that urogenital infections are often due to vaginal sex while anorectal infections are infrequently due to anal sex.

- Autoinoculation between the female urogenital and anorectal anatomic location might play a role in sustaining high chlamydia prevalence.

- A shift to more anorectal testing of female STI clinic attendees might be considered for its (albeit modest) impact on reducing prevalence.

\author{
Handling editor Jackie A Cassell \\ Acknowledgements The authors thank Pippa Scott for commenting on the final \\ version.
}

Contributors JCMH designed the study, developed the mathematical model, performed the model analyses and drafted the manuscript. GAFSVL, NHTMD-M and CJPAH collected and analysed the data. NHTMDM and BHBvB contributed to the design of the study and JAB to the design of the mathematical model. All authors contributed to the interpretation of the results and commented on the manuscript.

Competing interests None declared.

Patient consent Obtained.

Ethics approval The study was approved by the Medical Ethics Committee of Maastricht University (11-4-108).

Provenance and peer review Not commissioned; externally peer reviewed.

Open Access This is an Open Access article distributed in accordance with the Creative Commons Attribution Non Commercial (CC BY-NC 4.0) license, which permits others to distribute, remix, adapt, build upon this work non-commercially, and license their derivative works on different terms, provided the original work is properly cited and the use is non-commercial. See: http://creativecommons.org/ licenses/by-nc/4.0/

\section{REFERENCES}

1 European Centre for Disease Prevention and Control (ECDC). Annual Epidemiological Report 2013. Reporting on 2011 surveillance data and 2012 epidemic intelligence data. Stockholm: ECDC, 2013.

2 Dukers-Muijrers NH, Schachter J, van Liere GA, et al. What is needed to guide testing for anorectal and pharyngeal Chlamydia trachomatis and Neisseria gonorrhoeae in women and men? Evidence and opinion. BMC Infect Dis 2015;15:533.

3 The Dutch College of General Practitioners (NHG). Het SOA-consult [In Dutch]. http://www.nhg.org/standaarden/samenvatting/het-soa-consult\#idm8544768 (accessed 2 Nov 2015).

4 Centers for Disease Control and Prevention (CDC). Sexually transmitted diseases treatment guidelines, 2015. MMWR Recomm Rep 2015;64:1-137.

5 Nwokolo NC, Dragovic B, Patel S, et al. 2015 UK national guideline for the management of infection with Chlamydia trachomatis. Int J STD AIDS 2016;27:251-67.

6 van Liere GA, Hoebe CJ, Wolffs PF, et al. High co-occurrence of anorectal chlamydia with urogenital chlamydia in women visiting an STI clinic revealed by routine universal testing in an observational study; a recommendation towards a better anorectal chlamydia control in women. BMC Infect Dis 2014;14:274.

7 Ostergaard L, Agner T, Krarup E, et al. PCR for detection of Chlamydia trachomatis in endocervical, urethral, rectal, and pharyngeal swab samples obtained from patients attending an STD clinic. Genitourin Med 1997;73:493-7.

8 Peters RP, Dubbink JH, van der Eem L, et al. Cross-sectional study of genital, rectal, and pharyngeal Chlamydia and gonorrhea in women in rural South Africa. Sex Transm Dis 2014;41:564-9.

9 Australian Sexual Health Alliance (ASHA). Australian STI management guidelines for use in primary care. http://www.sti.guidelines.org.au/ sexually-transmissible-infections/chlamydia (accessed 2 Nov 2015).

10 Geisler WM, Uniyal A, Lee JY, et al. Azithromycin versus doxycycline for urogenital Chlamydia trachomatis infection. N Engl J Med 2015;373:2512-21.

11 Kong FY, Tabrizi SN, Fairley CK, et al. The efficacy of azithromycin and doxycycline for the treatment of rectal chlamydia infection: a systematic review and meta-analysis. J Antimicrob Chemother 2015;70:1290-7.

12 Rank RG, Yeruva L. Hidden in plain sight: chlamydial gastrointestinal infection and its relevance to persistence in human genital infection. Infect Immun 2014;82:1362-71.

13 Craig AP, Kong FY, Yeruva L, et al. Is it time to switch to doxycycline from azithromycin for treating genital chlamydial infections in women? Modelling the impact of autoinoculation from the gastrointestinal tract to the genital tract. $B M C$ Infect Dis 2015;15:200.

14 Heijne JC, Herzog SA, Althaus $\mathrm{CL}$, et al. Insights into the timing of repeated testing after treatment for Chlamydia trachomatis: data and modelling study. Sex Transm Infect 2013;89:57-62.

15 Rutgers. Kerncijfers leefstijlmonitor seksuele gezondheid [In Dutch]. http://www. rutgers.nl/sites/rutgersnl/files/PDF/Kerncijfers\%20_leefstij/monitor_seksuele_ gezondheid_2014_0.pdf (accessed 9 Dec 2016).

16 Herzog SA, McClean H, Carne CA, et al. Variation in partner notification outcomes for chlamydia in UK genitourinary medicine clinics: multilevel study. Sex Transm Infect 2011;87:420-5.

17 Rijksinstituut voor Volksgezondheid en Milieu (RIVM). LCI-richtlijn Chlamydia trachomatis en lymfogranuloma venereum [In Dutch]. http://www.rivm.nl/ Documenten_en_publicaties/Professioneel_Praktisch/Richtlijnen/Infectieziekten/LCI_ richtlijnen/LCI_richtlijn_Chlamydia_trachomatis_en_lymfogranuloma_venereum (accessed 9 Dec 2016)

18 van Liere GA, Dirks JA, Hoebe CJ, et al. Anorectal Chlamydia trachomatis load is similar in men who have sex with men and women reporting anal sex. PLOS ONE 2015;10:e0134991.

19 Bogaards JA, Kretzschmar M, Xiridou M, et al. Sex-specific immunization for sexually transmitted infections such as human papillomavirus: insights from mathematical models. PLoS Med 2011:8:e1001147.

20 Heijne $J C$, Herzog SA, Althaus $C L$, et al. Case and partnership reproduction numbers for a curable sexually transmitted infection. J Theor Biol 2013;331: 38-47.

21 Hui B, Fairley CK, Chen $\mathrm{M}$, et al. Oral and anal sex are key to sustaining gonorrhoea at endemic levels in MSM populations: a mathematical model. Sex Transm Infect 2015;91:365-9.

22 Mooij SH, van Santen DK, Geskus RB, et al. The effect of HIV infection on anal and penile human papillomavirus incidence and clearance: a cohort study among MSM. AIDS 2016;30:121-32

23 Althaus $\mathrm{CL}$, Heijne JC, Low $\mathrm{N}$. Towards more robust estimates of the transmissibility of Chlamydia trachomatis. Sex Transm Dis 2012;39:402-4.

24 Lapides J. Mechanisms of urinary tract infection. Urology 1979;14:217-25.

25 Götz HM, van den Broek IV, Hoebe CJ, et al. High yield of reinfections by home-based automatic rescreening of Chlamydia positives in a large-scale register-based screening programme and determinants of repeat infections. Sex Transm Infect 2013:89:63-9.

26 Lau A, Bradshaw CS, Lewis D, et al. The efficacy of azithromycin for the treatment of genital Mycoplasma genitalium: a systematic review and meta-analysis. Clin Infect Dis 2015;61:1389-99.

27 Althaus CL, Heijne JCM, Roellin A, et al. Transmission dynamics of Chlamydia trachomatis affect the impact of screening programmes. Epidemics 2010;2: 123-31.

28 Xiridou M, Vriend HJ, Lugner AK, et al. Modelling the impact of chlamydia screening on the transmission of HIV among men who have sex with men. BMC Infect Dis 2013;13:436.

29 de Graaf H, Kruijer H, Acker J, et al. Seks onder je 25e 2: seksuele gezondheid van jongeren in Nederland anno 2012 [In Dutch]. Delft, Eburon Academic Publishers 2012.

30 Kong FY, Tabrizi SN, Law M, et al. Azithromycin versus doxycycline for the treatment of genital Chlamydia infection: a meta-analysis of randomized controlled trials. Clin Infect Dis 2014;59:193-205. 